Pacific

Journal of

Mathematics

FINITE GROUPS OF LIE TYPE OF SMALL RANK

Frauke M. Bleher

Volume $187 \quad$ No. 2

February 1999 


\title{
FINITE GROUPS OF LIE TYPE OF SMALL RANK
}

\author{
Frauke M. Bleher
}

\begin{abstract}
In this paper, it is proven that a conjecture of Zassenhaus is valid for all finite simple groups of Lie type of rank 1 and of rank 2 which are not of type ${ }^{2} A_{3}$ or ${ }^{2} A_{4}$. In particular, this conjecture holds for all finite simple groups with abelian Sylow 2-subgroups.
\end{abstract}

\section{Introduction.}

One of the key problems in the representation theory of finite groups is to represent a given finite group $G$ in a suitable way. To do so, invariants which classify $G$ up to isomorphisms are determined. One of the questions derived in this context is the so-called Isomorphism Problem (IP), which asks whether the existence of an isomorphism between the integral group algebras $\mathbb{Z} G$ and $\mathbb{Z} H$ implies that $G$ and $H$ are isomorphic. In the seventies, Zassenhaus conjectured a stronger version of (IP) describing the structure of the group of units $V(\mathbb{Z} G)$ of $\mathbb{Z} G$ with augmentation 1 :

(ZC). Any two subgroups $X, Y$ of $V(\mathbb{Z} G)$ which have the same order as $G$ are conjugate by a unit of $\mathbb{Q} G$.

The conjecture (ZC) is not true in general, as was shown by Roggenkamp and Scott who constructed a metabelian counter-example [38, IX §1]. Note that since this counter-example is a metabelian group, (IP) is still valid for this group. Recently, Hertweck also found a counter-example to the general validity of (IP) which is a soluble group [18].

Nevertheless, it still remains an interesting question for which classes of finite groups (ZC) is valid. Roggenkamp and Scott showed that (ZC) is valid for groups whose generalized Fitting subgroup is a $p$-group [37]. Moreover, Weiss was able to show that for nilpotent groups each finite subgroup of $V(\mathbb{Z} G)$ is conjugate to a subgroup of $G$ by a unit of $\mathbb{Q} G[45,46]$. In case $G$ is soluble, Cech style cohomology sets can be used to get obstructions for (ZC) to be true [30].

For finite simple groups however, new methods have to be developed to examine (ZC). In [4] it is proven that (ZC) is valid for all minimal simple groups and for all simple Zassenhaus groups. But it is still an open question whether the conjecture (ZC) is valid for all finite simple groups. 
If $G$ is finite simple, then (IP) has always a positive solution [29, Thm. 2.3]. Furthermore, from [5, Thm. 1] it follows that (IP) is valid for all finite groups of Lie type $\mathbb{G}^{F}$, where $\mathbb{G}$ is a simply connected simple algebraic group over an algebraically closed field of finite characteristic and $F$ is a Frobenius map in the sense of $[8, \S 1.17]$. Note that these groups $\mathbb{G}^{F}$ are certain central extensions of the finite simple groups of Lie type. In general, if (IP) holds for $G,(\mathrm{ZC})$ is equivalent to the following description of the group $\operatorname{Aut}_{n}(\mathbb{Z} G)$ of augmentation preserving ring automorphisms of $\mathbb{Z} G$ :

(ZCAut). Every $\sigma \in \operatorname{Aut}_{n}(\mathbb{Z} G)$ can be written as $\sigma=\tau \circ \alpha$, where $\alpha$ is the $\mathbb{Z}$-linear extension of a group automorphism of $G$ and $\tau \in \operatorname{Aut}_{n}(\mathbb{Z} G)$ fixes the class sums of $G$.

Thus, if (IP) is valid for $G$, the structure of certain ring automorphisms of $\mathbb{Z} G$ can be used to study the group of units $V(\mathbb{Z} G)$. Since (IP) has a positive solution for all groups considered in this paper, we will always examine (ZCAut) to verify (ZC).

The main results of this paper are the following two theorems:

Theorem 1. The conjecture (ZC) is valid for all finite simple groups with abelian Sylow 2-subgroups.

Theorem 2. The conjecture (ZC) is valid for all finite simple groups of Lie type of rank 1 and of rank 2, which are not isomorphic to the unitary groups $\operatorname{PSU}\left(4, q^{2}\right)$ and $\operatorname{PSU}\left(5, q^{2}\right)$.

Note that because of the close relationship of the ordinary and modular representation theory of linear and unitary groups (see [26] and [43]), $\operatorname{PSU}\left(4, q^{2}\right)$ and $\operatorname{PSU}\left(5, q^{2}\right)$ should be examined together with the groups $\operatorname{PSL}(4, q)$ and $\operatorname{PSL}(5, q)$.

To prove these statements we use ordinary and modular representation theory. In particular, modular representations in the defining characteristic as described by Steinberg's tensor product theorem [43] play an important role. Here the knowledge of the action of $\sigma \in \operatorname{Aut}_{n}(\mathbb{Z} G)$ on tensor products as given in [4, Prop. 2.1] is essential. Furthermore, we need to examine the generic ordinary character tables of the considered groups. Except for the groups ${ }^{2} F_{4}\left(q^{2}\right)$, these character tables can be found in [9], [13], [14], $[\mathbf{1 5}],[\mathbf{1 6}],[34],[41],[42]$, or [44]. All character tables are also given in the computer algebra program CHEVIE [17] which provides generic ordinary character tables for several series of finite groups of Lie type of small rank. Furthermore, in CHEVIE known character tables have been verified, mistakes have been corrected and the data has been completed.

The article is organized as follows: In Section 2, we state basic results and techniques which are needed to prove the theorems. In particular, we show that if $G$ is a finite simple group of Lie type with defining characteristic $p$, or a certain central extension, then the action of $\sigma \in \operatorname{Aut}_{n}(\mathbb{Z} G)$ on the 
$p$-modular character table commutes with the operation induced by field automorphisms. In Section 3, the validity of (ZC) for the finite Ree groups of type $F_{4}$ and $G_{2}$ is proved. This establishes, together with the results of [4], Theorem 1. Section 4 is then dedicated to the proof of Theorem 2. Note that in [4] it is already shown that (ZC) is valid for the groups $\operatorname{SL}(2, q)$, $\operatorname{PSL}(2, q)$ and ${ }^{2} B_{2}\left(q^{2}\right)$ for all possible prime powers $q$.

The notations used are mainly standard, see for example [11]. The set of the conjugacy classes of $G$ is denoted by $\mathrm{Cl}(G), \mathrm{Cl}(g)$ is the conjugacy class of $g \in G$, and $\mathrm{Cl}\left(G_{p^{\prime}}\right)$ denotes the set of the conjugacy classes of $p$-regular elements of $G$. Suppose $(K, R, k)$ is a $p$-modular system with $K$ sufficiently large relative to $G$ and $\operatorname{char}(K)=0$. Thus both $K$ and $k$ are splitting fields for $G$. Then the set of the ordinary irreducible characters $\operatorname{Irr}(G)$ is identified with the set of the characters afforded by simple $K G$-modules. The irreducible Brauer characters $\operatorname{IBr}(G)$ are the irreducible Brauer characters with respect to $(K, R, k)$.

\section{Preliminaries.}

In this section, we want to provide basic results and techniques needed to prove the Theorems 1 and 2 .

Suppose $G$ is an arbitrary finite group and $(K, R, k)$ is a $p$-modular system with $K$ sufficiently large relative to $G$. Given $\sigma \in \operatorname{Aut}_{n}(\mathbb{Z} G), \sigma$ induces augmentation preserving algebra automorphisms $\sigma \in \operatorname{Aut}_{n}(K G)$, respectively $\sigma \in \operatorname{Aut}_{n}(k G)$, because there are ring homomorphisms from $\mathbb{Z}$ to $K$, respectively $k$. Then $\sigma$ defines an autoequivalence of the category of finitely generated $K G$-modules, respectively $k G$-modules, by mapping a module $M$ to $M^{\sigma}$. The twisted module $M^{\sigma}$ is defined to be equal to $M$ as vector space, but $g \in G$ operates on $M^{\sigma}$ as $\sigma(g)$. Thus $\sigma$ induces an operation on the ordinary characters, respectively Brauer characters, of $G$. Because $\sigma$ also induces a class sum correspondence $[38, I V]$, i.e. $\sigma$ maps class sums to class sums, $\sigma$ defines also an action on the conjugacy classes of $G$. These two actions are compatible in the sense that $\sigma$ induces a character table automorphism of the ordinary, respectively $p$-modular, character table of $G$. This means that for all $\chi \in \operatorname{Irr}(G), \varphi \in \operatorname{IBr}(G), C \in \mathrm{Cl}(G)$ and $C^{\prime} \in \mathrm{Cl}\left(G_{p^{\prime}}\right)$

$$
\begin{aligned}
\chi^{\sigma}(C) & =\chi\left(C^{\sigma}\right) \\
\varphi^{\sigma}\left(C^{\prime}\right) & =\varphi\left(C^{\prime \sigma}\right) .
\end{aligned}
$$

Furthermore, the operation of $\sigma$ on characters commutes with tensor products:

Proposition 2.1 ([4, Prop. 2.1]). Let $\xi$ and $\zeta$ be two ordinary characters, respectively Brauer characters, then

$$
(\xi \otimes \zeta)^{\sigma}=\xi^{\sigma} \otimes \zeta^{\sigma} .
$$


By [28, Thm. V.1(c)], it follows that $\sigma$ preserves also the power map in the sense that

$$
\left(C^{n}\right)^{\sigma}=\left(C^{\sigma}\right)^{n}
$$

for all positive integers $n$. Note that if $C=\mathrm{Cl}(g)$, then $C^{n}=\mathrm{Cl}\left(g^{n}\right)$.

The operation on the ordinary character table induced by $\sigma \in \operatorname{Aut}_{n}(\mathbb{Z} G)$ yields a statement which is equivalent to (ZCAut):

Lemma 2.2. The conjecture (ZCAut) is valid for $G$ if and only if for every $\sigma \in \operatorname{Aut}_{n}(\mathbb{Z} G)$ there exists $\alpha \in \operatorname{Aut}(G)$ such that $\sigma$ and $\alpha$ induce the same character table automorphism on the ordinary character table of $G$.

Thus Lemma 2.2 provides a necessary and sufficient criterion for the validity of (ZCAut) which uses only the ordinary character table and its automorphisms.

For the remainder of the paper, we want to concentrate on finite groups which are associated to Chevalley groups of universal type over an algebraically closed field.

Let $\mathcal{G}_{\mathbb{C}}$ be a simple Lie algebra over $\mathbb{C}$ with Cartan-subalgebra $\mathcal{H}_{\mathbb{C}}$. Let $\Phi$ be the root system of $\mathcal{G}_{\mathbb{C}}$ with respect to $\mathcal{H}_{\mathbb{C}}$, and let $\Delta=\left\{s_{1}, \ldots, s_{l}\right\}$ be the corresponding system of fundamental roots.

Suppose $\bar{k}$ is an algebraically closed field of characteristic $p>0$. The simply connected simple algebraic group, i.e. the Chevalley group of universal type, $\mathbb{G}$ of type $\Phi$ over $\bar{k}$ is generated by $\left\{x_{r}(t) \mid r \in \Phi, t \in \bar{k}\right\}$ with relations as in [7, Thm. 12.1.1]. If $h_{s}(t)=x_{s}(t) x_{-s}\left(-t^{-1}\right) x_{s}(t) x_{s}(-1) x_{-s}(1) x_{s}(-1)$, then $\mathbb{T}=\left\langle h_{s}(t) \mid s \in \Delta, t \in \bar{k}\right\rangle$ is a maximal torus of $\mathbb{G}$.

Since $\mathbb{G}$ is simply connected, the group $X=X(\mathbb{T})$ of rational characters of $\mathbb{T}$ is the full lattice of weights, with a basis consisting of the fundamental weights $\left\{\lambda_{1}, \cdots, \lambda_{l}\right\}$. Denote by $X^{+}$the set $\sum \mathbb{Z}_{0}^{+} \lambda_{i}$ of all dominant weights. There exists a natural partial ordering of $X$ given by $\mu \leq \lambda$ if $\lambda-\mu$ is a sum of positive roots. The group $X / p X$ of restricted weights with respect to $p$ is identified with $X_{p}=\left\{\sum c_{i} \lambda_{i} \mid 0 \leq c_{i} \leq p-1\right\}$. Given $\lambda \in X^{+}, \lambda$ can be written uniquely as $\lambda=\mu_{0}+p \mu_{1}+\cdots+p^{m-1} \mu_{m-1}$ for a suitable $m \geq 1$ with $\mu_{i} \in X_{p}$. If $X_{q}=\left\{\sum c_{i} \lambda_{i} \mid 0 \leq c_{i} \leq q-1\right\}, q=p^{m}$, then it follows that $\lambda \in X_{q}$.

Since $\mathcal{G}_{\mathbb{C}}$ has a Chevalley basis $\left\{e_{r}, h_{s} \mid r \in \Phi, s \in \Delta\right\}$ as $\mathbb{C}$-basis whose structure constants all lie in $\mathbb{Z}$, we can define $\mathcal{G}_{\mathbb{Z}}$ to be the $\mathbb{Z}$-lattice spanned by the Chevalley basis, and $\mathcal{G}_{\bar{k}}=\bar{k} \otimes_{\mathbb{Z}} \mathcal{G}_{\mathbb{Z}}$. Then $\mathcal{G}_{\bar{k}}$ is a Lie algebra over $\bar{k}$, and we denote $1 \otimes e_{r}$ and $1 \otimes h_{s}$ again by $e_{r}$ and $h_{s}$. A $\mathcal{G}_{\bar{k}}$-module $M$ is called restricted, if $e_{r}^{p} \cdot m=0$ and $h_{s}^{p} \cdot m=h_{s} \cdot m$ for all $r \in \Phi, s \in \Delta$ and $m \in M$.

The non-isomorphic irreducible restricted $\mathcal{G}_{\bar{k}}$-modules, which were described by Curtis (see $[\mathbf{4 3},(2.7)]$ ), correspond bijectively to the restricted weights in $X_{p}$. Let $\mathcal{M}$ be a full set of representatives of these modules, and 
let $M_{\lambda}$ denote the irreducible restricted $\mathcal{G}_{\bar{k}}$-module in $\mathcal{M}$ which corresponds to $\lambda \in X_{p}$.

According to $[43, \S 4]$ the $\mathcal{G}_{\bar{k}}$-module $M_{\lambda}$ can be lifted to a simple $\bar{k} \mathbb{G}-$ module $M_{\lambda}$ which is generated by a maximal vector $v^{+}$of highest weight $\lambda$. This means that if we look at the weight spaces $M_{\lambda}(\mu)=\left\{m \in M_{\lambda} \mid x \cdot m=\right.$ $\mu(x) \cdot m$ for all $x \in \mathbb{T}\}, \mu \in X$, then $v^{+} \in M_{\lambda}(\lambda)$ and $x_{r}(t) \cdot v^{+}=v^{+}$for all $r \in \Phi^{+}$. For all $\mu \in X$ with $M_{\lambda}(\mu) \neq 0$, it follows that $\mu \leq \lambda$.

Note that each field automorphism $\alpha$ of $\bar{k}$ induces a group automorphism $\alpha$ of $\mathbb{G}$ by defining $\alpha\left(x_{r}(t)\right)=x_{r}(\alpha(t)), r \in \Phi, t \in \bar{k}$.

The isomorphism classes of simple $\bar{k} \mathbb{G}$-modules can now be described as follows:

Theorem 2.3 (Chevalley, Kostant, Steinberg, see [23, §2.1]). Let $\alpha_{i}$ be the field automorphism of $\bar{k}$ which is defined by $\alpha_{i}(t)=t^{p^{i}}$. Let $\lambda \in X^{+}$, such that $\lambda \in X_{q}$ for a suitable $q=p^{m}, \lambda=\mu_{0}+p \mu_{1}+\cdots+p^{m-1} \mu_{m-1}$ with $\mu_{i} \in X_{p}$. Then

$$
M_{\lambda}=M_{\mu_{0}}^{\alpha_{0}} \otimes_{\bar{k}} M_{\mu_{1}}^{\alpha_{1}} \otimes_{\bar{k}} \cdots \otimes_{\bar{k}} M_{\mu_{m-1}}^{\alpha_{m-1}}
$$

is a simple $\bar{k} \mathbb{G}$-module. All $M_{\lambda}\left(\lambda \in X^{+}\right)$are pairwise non-isomorphic and exhaust the isomorphism classes of simple $\bar{k} \mathbb{G}$-modules.

Let $q=p^{n}$ and let $F=\operatorname{GF}(q)$ be the finite subfield of $\bar{k}$ with $q$ elements. Then the finite Chevalley group $G=\mathbb{G}(F)$ over $F$ is generated by $\left\{x_{r}(t) \mid r \in \Phi, t \in F\right\}$ with the same relations as $\mathbb{G}$. Note: $\bar{G}=G / \operatorname{center}(G)$ is a simple group except for the cases $\operatorname{SL}(2,2), \operatorname{PSL}(2,3), \operatorname{Sp}(4,2)$ and $G_{2}(2)$ [7, Thm. 11.1.2].

The twisted groups are certain subgroups of finite Chevalley groups:

Suppose $G$ is a finite Chevalley group of type $A_{l}, l \geq 2, D_{l}, l \geq 4, E_{6}$, or $D_{4}$, respectively, over a finite field $F$. Let $\omega$ be a nontrivial symmetry of order $e$ of the Dynkin diagram and let $\gamma$ be the corresponding graph automorphism. Let $\alpha$ be a nontrivial field automorphism such that $\Omega=\gamma \alpha$ satisfies $\Omega^{e}=1$, and let $F_{0}$ be the subfield of $F$ fixed by $\alpha$. Note that $F$ is of the form $F=\operatorname{GF}\left(p^{n e}\right)$ and $F_{0}=\mathrm{GF}\left(p^{n}\right)$. If $G^{1}$ is defined to be the group of all elements of $G$ fixed by $\Omega$, then $G^{1}$ is a twisted group of type ${ }^{2} A_{l}, l \geq 2$, ${ }^{2} D_{l}, l \geq 4,{ }^{2} E_{6}$, or ${ }^{3} D_{4}$, respectively. $\bar{G}^{1}=G^{1} / \operatorname{center}\left(G^{1}\right)$ is simple except for $\operatorname{PSU}\left(3,2^{2}\right)[7$, Thm. 14.4.1].

The definition of the finite Suzuki and Ree groups is slightly different:

Suppose $G$ is a finite Chevalley group of type $B_{2}, G_{2}$, or $F_{4}$, respectively, over a finite field $F$ which has characteristic 2 for $B_{2}$ and $F_{4}$, and characteristic 3 for $G_{2}$. Let $\omega$ be the nontrivial symmetry of the Dynkin diagram with corresponding graph automorphism $\gamma$. Let $\alpha$ be a nontrivial field automorphism such that $\Omega^{2}=1$ for $\Omega=\gamma \alpha$. Note that $F$ is of the form $F=\mathrm{GF}\left(p^{2 m+1}\right), p=2$ or 3 . If $G^{1}$ is the group of all elements of $G$ fixed by 
$\Omega$, then $G^{1}$ is a Suzuki group if $G$ has type $B_{2}$, and a Ree group if $G$ has type $G_{2}$ or $F_{4}$. $G^{1}$ is simple for all positive $m$ [7, Thm. 14.4.1].

Since the $\bar{k} \mathbb{G}$-modules $M_{\lambda}, \lambda \in X_{q}$, define by restriction $\bar{k} G$-modules, respectively $\bar{k} G^{1}$-modules, we get the following theorem:

Theorem 2.4 ([43, Thm. 7.4, Thm. 9.3 and Thm. 12.2]). Let $G$ be a finite Chevalley group over $\mathrm{GF}\left(p^{n}\right)$, or a twisted group of type ${ }^{2} A_{l}, l \geq 2$, ${ }^{2} D_{l}, l \geq 4,{ }^{2} E_{6}$, or ${ }^{3} D_{4}$ over $\mathrm{GF}\left(p^{n e}\right)$ where $e$ is the order of the symmetry of the corresponding Dynkin diagram. Then every simple $\bar{k} G-$ module can be expressed uniquely as a tensor product

$$
M_{0}^{\alpha_{0}} \otimes_{\bar{k}} M_{1}^{\alpha_{1}} \otimes_{\bar{k}} \cdots \otimes_{\bar{k}} M_{n-1}^{\alpha_{n-1}} \quad \text { with } M_{i} \in \mathcal{M} .
$$

In case that $G$ is a Suzuki or Ree group of type ${ }^{2} B_{2},{ }^{2} G_{2}$, or ${ }^{2} F_{4}$ over $\operatorname{GF}\left(p^{n}\right)$, $p=2$ or 3 , set $X_{p}^{\prime}=\left\{\lambda=\sum c_{i} \lambda_{i} \in X_{p} \mid c_{i}=0\right.$ for all long roots $\left.s_{i} \in \Delta\right\}$ and $\mathcal{M}^{\prime}=\left\{M_{\lambda} \mid \lambda \in X_{p}^{\prime}\right\}$. Then every simple $\bar{k} G$-module can be expressed uniquely as a tensor product

$$
M_{0}^{\alpha_{0}} \otimes_{\bar{k}} M_{1}^{\alpha_{1}} \otimes_{\bar{k}} \cdots \otimes_{\bar{k}} M_{n-1}^{\alpha_{n-1}} \quad \text { with } M_{i} \in \mathcal{M}^{\prime} .
$$

Remark 2.5. Let $G$ be a finite Chevalley group, a twisted group or a finite Suzuki or Ree group.

(i) Since the simple $\bar{k} G$-modules are given as restrictions of certain simple modules of the corresponding Chevalley group $\mathbb{G}$ of universal type, we can attach weights $\lambda \in X^{+}$to the simple $\bar{k} G$-modules. If we choose $\lambda \in X_{p^{n}}, n$ as in Theorem 2.4, then these weights determine the simple $\bar{k} G$-modules uniquely up to isomorphisms. In this sense we will write the simple $\bar{k} G$-modules also as $M_{\lambda}$ for suitable weights $\lambda \in X_{p^{n}}$. Furthermore, the Brauer character corresponding to $M_{\lambda}$ will be denoted by $\beta_{\lambda}$.

(ii) We will also use $M_{\lambda}$ to denote the $\bar{k} G$-module obtained by restriction of the $\bar{k} \mathbb{G}$-module $M_{\lambda}$ in case $\lambda \in X^{+} \backslash X_{q}$. Note that then this restriction is not necessarily a simple $\bar{k} G$-module. Again the corresponding Brauer character is denoted by $\beta_{\lambda}$.

(iii) The simple modules for the groups $\bar{G}=G / \operatorname{center}(G)$ are exactly those simple $\bar{k} G$-modules on which the center acts trivially.

(iv) For the finite groups the algebraically closed field $\bar{k}$ can be replaced by any splitting field of the same characteristic.

We want now to describe some general properties of the considered finite groups of Lie type which are also important for the proofs of the Theorems 1 and 2.

For the remainder of the paper, we fix the following notation:

Let $G$ be a finite Chevalley group over $\operatorname{GF}\left(p^{n}\right)$, a twisted group over $\operatorname{GF}\left(p^{n e}\right)$, or a finite Suzuki or Ree group over $\mathrm{GF}\left(p^{n}\right)$ as in Theorem 2.4, and let $q=p^{n}$. Let $Z=\operatorname{center}(G)$ and let $\bar{G}=G / Z$. The group automorphism 
of $G$ induced by the field automorphism $t \mapsto t^{p^{i}}$ will be denoted by $\alpha_{i}$. Then $\bar{\alpha}_{i}$ with $\bar{\alpha}_{i}(g Z)=\alpha_{i}(g) Z$ is the corresponding group automorphism of $\bar{G}$. Let $\sigma \in \operatorname{Aut}_{n}(\mathbb{Z} G)$ and $\bar{\sigma} \in \operatorname{Aut}_{n}(\mathbb{Z} \bar{G})$ be arbitrary elements.

Let $(K, R, k)$ be a $p$-modular system with $K$ sufficiently large relative to $G$ such that $\bar{k}$ is an algebraic closure of $k$. Since $k$ is a splitting field for $G$, the isomorphism classes of simple $k G$-modules can be identified with those of simple $\bar{k} G$-modules. Let $\mathbb{G}$ be the Chevalley group of universal type over $\bar{k}$ corresponding to $G$.

Lemma 2.6. The action of $\sigma$ on the simple $k G$-modules $S$ commutes with the action of $\alpha_{i}$ for all $i$, i.e. $S^{\sigma \alpha_{i}} \cong S^{\alpha_{i} \sigma}$ for all $i$. The action of $\alpha_{i}$ on the $p$-regular conjugacy classes of $G$ is given by $\mathrm{Cl}(g) \mapsto \mathrm{Cl}\left(g^{p^{i}}\right)$.

Proof. Because of Proposition 2.1 and Theorem 2.4, it suffices to show this for the simple modules $M \in \mathcal{M}$ or $\mathcal{M}^{\prime}$, respectively. Consider $M$ as a $\bar{k} G$-module. Thus we have to show $M^{\alpha_{i} \sigma} \cong M^{\sigma \alpha_{i}}$ as $\bar{k} G$-modules.

Suppose $\rho: G \rightarrow \mathrm{GL}(m, \bar{k})$ is the representation corresponding to $M$ as a $\bar{k} G$-module. Let $\nu_{i}$ be the automorphism of $\operatorname{GL}(m, \bar{k})$, which maps a matrix $\left(a_{r s}\right)$ to $\left(a_{r s}^{p^{i}}\right)$. Then, if $M^{(i)}$ denotes the module corresponding to the representation $\nu_{i} \circ \rho$, we get $M^{\alpha_{i}} \cong M^{(i)}$ as $\bar{k} G$-modules (see for example [31, Prop. 5.4.2.(i)]). A simple matrix calculation shows that, if $\xi_{1}, \ldots, \xi_{m}$ are the eigenvalues of $\rho(g)$, then $\xi_{1}^{p^{i}}, \ldots, \xi_{m}^{p^{i}}$ are the eigenvalues of $\nu_{i}(\rho(g))$ and of $\rho\left(g^{p^{i}}\right)$.

If $\varphi$ is the Brauer character corresponding to $\rho$, and $\varphi^{(i)}$ is the Brauer character corresponding to $\nu_{i} \circ \rho$, then it follows that

$$
\varphi^{\alpha_{i}}(g)=\varphi^{(i)}(g)=\varphi\left(g^{p^{i}}\right)
$$

for all $p$-regular elements $g \in G$.

Since Equation (2.1) is valid for all Brauer characters of $G$ by Proposition 2.1, and since $g \mapsto g^{p^{i}}$ is a permutation of the $p$-regular elements, the permutation of the $p$-regular conjugacy classes of $G$ corresponding to $\alpha_{i}$ is given by $\mathrm{Cl}(g) \mapsto \mathrm{Cl}\left(g^{p^{i}}\right)$.

Since $\sigma$ preserves the power map, i.e. $\left(C^{a}\right)^{\sigma}=\left(C^{\sigma}\right)^{a}$ for all conjugacy classes $C=\mathrm{Cl}(g)$ and $C^{a}=\mathrm{Cl}\left(g^{a}\right)$, it follows that $\varphi^{\alpha_{i} \sigma}=\varphi^{\sigma \alpha_{i}}$ and therefore, $M^{\alpha_{i} \sigma} \cong M^{\sigma \alpha_{i}}$ for all $M \in \mathcal{M}$ or $\mathcal{M}^{\prime}$, respectively.

Corollary 2.7. The operation of $\bar{\alpha}_{i}$ on the p-regular conjugacy classes of $\bar{G}$ is given by $\mathrm{Cl}(\bar{g}) \mapsto \mathrm{Cl}\left(\bar{g}^{p^{i}}\right)$. In particular, it follows that $S^{\bar{\sigma} \bar{\alpha}_{i}} \cong S^{\bar{\alpha}_{i} \bar{\sigma}}$ for all simple $k \bar{G}$-modules $S$.

Proof. By Lemma 2.6, $\alpha_{i}(\mathrm{Cl}(g))=\mathrm{Cl}\left(g^{p^{i}}\right)$, i.e. $\alpha_{i}(g)$ is conjugate to $g^{p^{i}}$ in $G$ for all $p$-regular elements $g \in G$. Therefore, $\bar{\alpha}_{i}(g Z)$ is conjugate to $(g Z)^{p^{i}}$ in $\bar{G}$, i.e. $\bar{\alpha}_{i}(\bar{g})$ is conjugate to $\bar{g}^{p^{i}}$ for all $p$-regular elements $\bar{g} \in \bar{G}$. Thus we have that $\bar{\alpha}_{i}(\mathrm{Cl}(\bar{g}))=\mathrm{Cl}\left(\bar{g}^{p^{i}}\right)$. Because $\bar{g} \mapsto \bar{g}^{p^{i}}$ is a permutation 
of the $p$-regular elements of $\bar{G}$ and because $\bar{\sigma}$ preserves the power map, the corollary follows.

With respect to the weights of tensor products we have the following result.

Lemma 2.8. Let $\lambda, \mu \in X^{+}$, and let $M_{\lambda}$ and $M_{\mu}$ be the two (not necessarily simple) $k G$-modules which are restrictions of the corresponding simple $\bar{k} \mathbb{G}$ modules. Then $M_{\lambda+\mu}$ is a factor module of $M_{\lambda} \otimes M_{\mu}$ as $k G$-modules. Furthermore, as $\bar{k} \mathbb{G}$-modules, $\lambda+\mu$ is the highest weight occurring in $M_{\lambda} \otimes M_{\mu}$.

Suppose now that $M_{\lambda}$ and $M_{\mu}$ are simple $k G$-modules with $\lambda, \mu \in X_{q}$. If $M_{\lambda} \otimes M_{\mu}$ is also a simple $k G$-module then $M_{\lambda} \otimes M_{\mu} \cong M_{\lambda+\mu}$ as $k G$-modules. Since $\lambda+\mu \in X_{p q}$, it follows that $\lambda+\mu=\nu_{0}+q \nu_{1}$ for appropriate $\nu_{0} \in X_{q}$ and $\nu_{1} \in X_{p}$. Thus

$$
M_{\lambda+\mu} \cong M_{\nu_{0}} \otimes M_{\nu_{1}}^{\alpha_{n}}
$$

as $k G$-modules. Note that $M_{\nu_{1}}^{\alpha_{n}} \cong M_{\nu_{1}}$ if $G$ is a finite Chevalley group or a Suzuki or Ree group. Otherwise $M_{\nu_{1}}^{\alpha_{n}} \cong M_{\omega^{-1}\left(\nu_{1}\right)}$ where $\omega$ is the symmetry of the Dynkin diagram in the definition of the twisted groups.

Proof. Let $\lambda, \mu \in X^{+}$. Then there exist maximal vectors $v$, respectively $w$, of highest weight $\lambda$, respectively $\mu$, in the $\bar{k} \mathbb{G}$-module $M_{\lambda}$, respectively $M_{\mu}$. By $[\mathbf{2 2}, \S 31.4], v \otimes w$ is then a maximal vector of the $\bar{k} \mathbb{G}$-module $M_{\lambda} \otimes M_{\mu}$ of weight $\lambda+\mu$, which is the highest weight occurring in $M_{\lambda} \otimes M_{\mu}$. So $M_{\lambda+\mu}$ is a factor module of $M_{\lambda} \otimes M_{\mu}$ as $\bar{k} \mathbb{G}$-modules and thus as $k G$-modules.

Let now $\lambda, \mu \in X_{q}$. If $M_{\lambda} \otimes M_{\mu}$ is simple as a $k G$-module then $M_{\lambda} \otimes M_{\mu}$ is also simple as a $\bar{k} \mathbb{G}$-module. Thus $M_{\lambda} \otimes M_{\mu}$ is simple with weight $\lambda+\mu$. Since $\lambda, \mu \in X_{q}$ and $2(q-1) \leq p q-1$, it follows that $\lambda+\mu=\nu_{0}+q \nu_{1}$ for appropriate $\nu_{0} \in X_{q}$ and $\nu_{1} \in X_{p}$. Thus $M_{\lambda+\mu} \cong M_{\nu_{0}} \otimes_{k} M_{\nu_{1}}^{\alpha_{n}}$ as $k G$-modules.

We want now to outline the strategy to prove the conjecture (ZC) for certain $G$ and $\bar{G}$.

Definition 2.9. Denote by $\bar{X}^{+}$all dominant weights $\lambda \in X^{+}$such that $M_{\lambda}$ is a $k \bar{G}$-module. In case that $G$ is a Suzuki or a Ree group, let $X^{+^{\prime}}=$ $\left\{\lambda=\sum c_{i} \lambda_{i} \in X^{+} \mid c_{i}=0\right.$ for all long roots $\left.s_{i} \in \Delta\right\}$. Let $Y^{+}$be either $X^{+}, X^{+^{\prime}}$, or $\bar{X}^{+}$.

(i) A relation $\prec$ on $Y^{+}$is called a weak order if $\prec$ is reflexive and transitive.

(ii) An allowable system on $Y^{+}$is a subset $R \subseteq Y^{+}$such that all $\lambda \in Y^{+}$ can be written as

$$
\lambda=\sum_{\rho \in R} d_{\rho} \rho
$$

for a unique choice of integers $d_{\rho} \geq 0$. 
(iii) Let $R$ be an allowable system and $\prec$ a weak order on $Y^{+}$. Then $\prec$ is called an allowable weak order if the following properties are fulfilled:

(a) If $\lambda \in\{0\} \cup R$ and $\mu \in Y^{+}$with $\mu \prec \lambda$, then $\mu \in\{0\} \cup R$.

(b) If $\mu_{1} \leq \mu_{2}$ and $\mu_{1} \neq \mu_{2}$, it follows that $\mu_{1} \prec \mu_{2}$ and $\mu_{2} \nprec \mu_{1}$.

(c) If $\lambda=\sum d_{\rho} \rho$ is a dominant weight with $\lambda \notin\{0\} \cup R$, then there exists $\rho_{0} \in R$ with $\rho_{0} \prec \lambda$ and $\lambda-\rho_{0} \prec \lambda$, but $\lambda \nprec \lambda-\rho_{0}$.

Remark 2.10. (i) If $\prec$ is a weak order on $Y^{+}$then we can define an equivalence relation $\sim$ on $Y^{+}$by

$$
\lambda \sim \mu \Leftrightarrow \lambda \prec \mu \text { and } \mu \prec \lambda .
$$

Then $\prec$ defines a partial ordering of $Y^{+} / \sim$, the equivalence classes of $Y^{+}$with respect to $\sim$.

(ii) For $Y^{+}=X^{+}$(respectively $X^{+^{\prime}}$ ) we can and will always choose the fundamental weights $\lambda_{i} \in X_{p}$ (respectively $X_{p}^{\prime}$ ) as allowable system.

(iii) Definition 2.9 is written in this generality since we want to prove (ZC) for groups $G$ and $\bar{G}$.

(iv) The order relation $\leq$ on $X^{+}$does not always fulfill property (c). For example, for $\mathrm{SL}(3, q), \lambda_{1} \not \leq \lambda_{1}+\lambda_{2}$ and $\lambda_{2} \not \leq \lambda_{1}+\lambda_{2}$.

The following proposition plays an important role in the examination of (ZC).

Proposition 2.11. Let $\left(H, Y^{+}\right)$be either $\left(G, X^{+}\right),\left(G, X^{+^{\prime}}\right)$, or $\left(\bar{G}, \bar{X}^{+}\right)$, let $\tau$ be either $\sigma$ or $\bar{\sigma}$, and let $R$ be an allowable system of $Y^{+}$. Suppose

(i) there exists a group automorphism $\alpha \in \operatorname{Aut}(H)$ with $M_{\rho}^{\tau} \cong M_{\rho}^{\alpha}$ for all $\rho \in R$, and

(ii) there exists an allowable weak order $\prec$ on $Y^{+}$.

Then $\tau$ and $\alpha$ induce the same operation on the p-modular character table of $H$.

Proof. We have to show that $\beta_{\lambda}^{\tau}=\beta_{\lambda}^{\alpha}$ for all $\lambda \in Y^{+} \cap X_{q}$.

By (i) there exists a group automorphism $\alpha$ such that $\beta_{\rho}^{\tau}=\beta_{\rho}^{\alpha}$ for all $\rho \in R$. Thus, by property (a) of $\prec$, the statement is valid for the smallest weights with respect to $\prec$.

Let now $\lambda \in Y^{+}, \lambda \notin\{0\} \cup R$. Suppose $\beta_{\mu}^{\tau}=\beta_{\mu}^{\alpha}$ for all $\mu \in Y^{+}$with $\mu \prec \lambda$ and $\lambda \nprec \mu$, which means, by property (b) of $\prec$, in particular for all $\mu \in Y^{+}$with $\mu \leq \lambda, \mu \neq \lambda$. By property (c), there exists $\rho_{0} \in R$ such that $\mu_{1}=\rho_{0} \prec \lambda$, and $\mu_{2}=\lambda-\rho_{0} \prec \lambda$, but $\lambda \nprec \mu_{2}$. Thus $\beta_{\mu_{s}}^{\tau}=\beta_{\mu_{s}}^{\alpha}$ for $s \in\{1,2\}$. By Lemma 2.8, $\beta_{\mu_{1}} \otimes \beta_{\mu_{2}}$ can be written as

$$
\beta_{\mu_{1}} \otimes \beta_{\mu_{2}}=\sum_{\nu \in Y^{+}} a_{\nu} \beta_{\nu} \quad \text { with } a_{\nu} \geq 0
$$

such that $a_{\lambda} \neq 0$ and $\nu \leq \lambda$ for all other $\nu \in Y^{+}$with $a_{\nu} \neq 0$. Using Proposition 2.1, it follows that $\beta_{\lambda}^{\tau}=\beta_{\lambda}^{\alpha}$. 
Remark 2.12. (i) Proposition 2.11 implies that $\tau$ operates as $\alpha$ on all ordinary irreducible characters which are not $p$-exceptional. Note that a character $\chi \in \operatorname{Irr}(H)$ is called $p$-exceptional, if there exists another character $\chi^{\prime} \in \operatorname{Irr}(\mathrm{H})$ such that $\chi$ and $\chi^{\prime}$ have the same values on the $p$-regular classes of $H$.

(ii) All group automorphisms of $G$, respectively $\bar{G}$, are given as the composition of an inner, a diagonal, a graph and a field automorphism [7, Thm. 12.5.1]. Since the inner and diagonal automorphisms operate trivially on the $p$-regular classes, $\alpha$ can be chosen as the composition of a graph and a field automorphism.

Let $H, \tau$ and $\alpha$ be as in Proposition 2.11. Then $\tau \alpha^{-1}$ operates trivially on all ordinary irreducible characters which are not $p$-exceptional. We will use ad-hoc techniques to refine $\alpha$ to a group automorphism $\beta$ of $H$ such that $\tau \beta^{-1}$ fixes also all $p$-exceptional characters. Using Lemma 2.2 this then implies that (ZC) is valid for $H$.

In this context, $l$-blocks of $H$ which have cyclic defect groups and their corresponding Brauer trees are important. Note that usually the prime $l$ is different from $p$.

There are two interpretations of the Brauer tree corresponding to a block $B$ with cyclic defect groups. One approach is to define the Brauer tree by only using the category of finitely generated $B$-modules. This is described in $[\mathbf{1}$, Chapter V]. The other possibility is to define the Brauer tree using the decomposition map. This follows from Brauer's and Dade's theory of blocks with cyclic defect groups (see [12]). By $[\mathbf{1 2}$, Thm. 1] it follows that these two definitions coincide. As a reference for both interpretations of the Brauer tree of $B$ use for example [6].

With respect to the operation of $\tau$ on the Brauer trees corresponding to $l$-blocks with cyclic defect groups, we need the following two results. Note that $\tau$ permutes the $l$-blocks of $H$.

Lemma 2.13. Let $B$ be an l-block of $H$ with cyclic defect groups such that $\tau$ fixes $B$. Then $\tau$ induces a graph automorphism of the Brauer tree of $B$ which is either trivial or a rotation.

Proof. This follows from [6, Prop. 3.7] and the proof of [6, Cor. 3.9].

Lemma 2.14 ([6, Cor. 3.10]). Let $H$ have cyclic Sylow l-subgroups and let $B$ be the principal l-block. If $\chi \in \operatorname{Irr}(H)$ belongs to $B$ and if $\chi$ is not $l$ exceptional, then $\tau$ fixes $\chi$.

We use these two lemmas in the following way. To refine $\alpha$ of Proposition 2.11 , we want to show that $\tau \alpha^{-1}$ operates on the $p$-exceptional characters as the power of a diagonal automorphism. In case all diagonal automorphisms of $H$ are inner, we use Lemma 2.14 for certain $l$ to show that $\tau \alpha^{-1}$ fixes all $p$-exceptional characters. In the case of the Ree groups of type $F_{4}$, Lemma 
2.13 is used to eliminate operations on characters which are not induced by group automorphisms.

In case that $H$ has a nontrivial diagonal automorphism $\delta$, the characters permuted by $\delta$ have to be examined separately. Here we use, if necessary, Lemma 2.13 to study all possible operations on Brauer trees which contain such characters.

Remark 2.15. (i) To determine the $p$-exceptional characters we use mostly the generic character tables provided in CHEVIE [17]. As outlined in the introduction there are literature references for most of the considered tables, which are either verified or corrected in CHEVIE.

(ii) In the examination of the $p$-exceptional characters, these characters are decomposed into a union of pairwise disjoint subsets such that all characters which have the same values on the $p$-regular classes are in one such subset.

(iii) When Brauer trees are given, a black circle $\bullet$ denotes the exceptional vertex.

According to the above described strategy, the proofs of the validity of (ZC) for certain $G$ (respectively $\bar{G}$ ) will always be divided into two parts:

(A) Operation of $\sigma$ (respectively $\bar{\sigma}$ ) on the $p$-modular character table. Here we only have to check the hypotheses of Proposition 2.11.

(B) Operation of $\sigma$ (respectively $\bar{\sigma}$ ) on the $p$-exceptional characters.

Here we use ad-hoc arguments together with Lemmas 2.13 and 2.14.

The proofs involve the following Dynkin diagrams: $A_{2}, B_{2}, G_{2}, D_{4}, F_{4}$. 


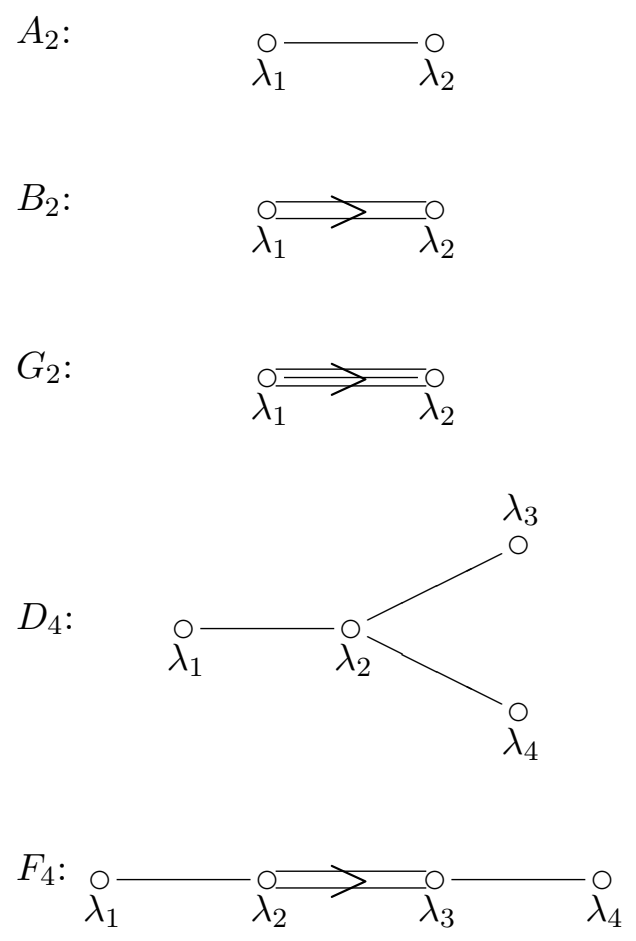

The arrow always points to the shorter roots.

A weight $\lambda=\sum c_{i} \lambda_{i}$ will be denoted by $\lambda=c_{1} \cdots c_{l}$.

\section{The Ree groups ${ }^{2} G_{2}\left(q^{2}\right)$ and ${ }^{2} F_{4}\left(q^{2}\right)$.}

In this section, we want to prove that $(\mathrm{ZC})$ is valid for the finite Ree groups of type $G_{2}$ and $F_{4}$, and thus establish Theorem 1.

The Ree groups ${ }^{2} G_{2}\left(q^{2}\right)$ and ${ }^{2} F_{4}\left(q^{2}\right)$ are simple except for ${ }^{2} G_{2}(3)$ and ${ }^{2} F_{4}(2)$; their automorphisms are given by inner and field automorphisms. The generic ordinary character table of ${ }^{2} G_{2}\left(3^{2 m+1}\right)$ was mostly determined by Ward in [44]. Shinoda described the conjugacy classes of ${ }^{2} F_{4}\left(2^{2 m+1}\right)$ in [40]. The complete ordinary character table of ${ }^{2} G_{2}\left(3^{2 m+1}\right)$ can be found in CHEVIE; most of the character table of ${ }^{2} F_{4}\left(2^{2 m+1}\right)$ and all Green functions are also listed there.

Proposition 3.1. (i) The conjecture (ZC) is valid for ${ }^{2} G_{2}\left(q^{2}\right)$ for all $q^{2}=3^{2 m+1}, m \geq 1$

(ii) The conjecture (ZC) is valid for ${ }^{2} F_{4}\left(q^{2}\right)$ for all $q^{2}=2^{2 m+1}, m \geq 1$.

Proof. Let $G={ }^{2} G_{2}\left(q^{2}\right)$ or $G={ }^{2} F_{4}\left(q^{2}\right)$, respectively. 
Part (A).

(i) $X_{p}^{\prime}=\{00,01,02\}$ with $\operatorname{dim}\left(M_{01}\right)=7$ and $\operatorname{dim}\left(M_{02}\right)=27$. Because of dimensions, there exists $0 \leq i \leq 2 m$ with $M_{01}^{\sigma} \cong M_{01}^{\alpha_{i}}$. Since $\leq$ defines an allowable weak order $\prec$ on $X^{+}$, the hypotheses of Proposition 2.11 are satisfied.

(ii) $X_{p}^{\prime}=\{0000,0010,0001,0011\}$ with $\operatorname{dim}\left(M_{0001}\right)=26, \operatorname{dim}\left(M_{0010}\right)=$ 246 and $\operatorname{dim}\left(M_{0011}\right)=4096$. Because of dimensions, there exist $0 \leq$ $i, j \leq 2 m$ with $M_{0001}^{\sigma} \cong M_{0001}^{\alpha_{i}}$ and $M_{0010}^{\sigma} \cong M_{0010}^{\alpha_{j}}$. We have to show that $i=j$. If $i \neq j$ then $\sigma$ maps the simple module $M_{0001}^{\alpha_{2 m+1-i}} \otimes$ $M_{0010}^{\alpha_{2 m+1-j}}$ to $M_{0001} \otimes M_{0010}$. This implies that $M_{0001} \otimes M_{0010}$ must be simple, thus by Lemma 2.8 of weight 0011. A comparison of the dimensions shows that this is impossible. Thus it follows that $i=j$ which yields hypothesis (i) of Proposition 2.11. $\leq$ defines again an allowable weak order $\prec$ on $X^{+}$, which is hypothesis (ii).

Part (B).

(i) $G$ does not have any nontrivial diagonal automorphisms, thus we have to show that $\sigma \alpha_{i}^{-1}, \alpha_{i}$ from Part (A), fixes all ordinary characters which are $p$-exceptional. The generic character table given in CHEVIE shows that there are exactly 6 unipotent characters which are $p$-exceptional: Using the notation of $[\mathbf{2 0}]$, these characters correspond to $\left\{\xi_{3}, \xi_{4}\right\},\left\{\xi_{5}, \xi_{6}\right\}$ and $\left\{\xi_{7}, \xi_{8}\right\}$. If $l>3$ is a rational prime which divides $|G|$, then $G$ has cyclic Sylow $l$-subgroups $[25$, XI Thm. 13.2]. By [20, Thm. 4.2], $\xi_{5}, \xi_{6}$ and $\xi_{7}, \xi_{8}$ lie in the principal $l$-block for $l \mid\left(q^{2}+1\right)$ and are not $l$-exceptional. By [20, Thm. 4.3], $\xi_{3}, \xi_{4}$ and $\xi_{5}, \xi_{6}$ lie in the principal $l$-block for $l \mid\left(q^{2}+\sqrt{3} q+1\right)$ and are not $l$ exceptional. Thus, by Lemma $2.14, \sigma \alpha_{i}^{-1}$ fixes all ordinary characters, which proves Proposition 3.1(i).

(ii) Since the generic character table of $G$ given in CHEVIE is not complete, we have to look at this case more carefully. Again we have to show that $\sigma \alpha_{i}^{-1}$ operates trivially on the characters which are $p$ exceptional.

Let $\mathbb{G}$ be the Chevalley group of universal type of type $F_{4}$ over $\bar{k}$, and let $F$ be the Frobenius map (notation used as in $[8, \S 1.17]$ ) with $\mathbb{G}^{F}=G$. Using the Jordan decomposition for characters, the ordinary irreducible characters of $G$ can be described as follows (cf. $[8, \S 12.9])$ : Since the Ree groups are self-dual, the characters of $G$ are parametrized by $G$-conjugacy classes of pairs $(s, \lambda)$ where $s$ is a semisimple element of $G$ and $\lambda$ is a unipotent character of $C_{G}(s)$. This character is then denoted by $\chi_{s, \lambda}$. Since $G$ is self-dual, the $G$-conjugacy classes of semisimple elements of $G$ correspond to geometric conjugacy classes. 
We use the notation in [40] for the 11 conjugacy classes of the maximal tori and the representatives of the semisimple conjugacy classes, i.e. of the semisimple class types. Note that the $G$-conjugacy classes of the maximal tori $\mathbb{T}$ of $\mathbb{G}$ are uniquely determined by the isomorphism type of $\mathbb{T}^{F}$.

In CHEVIE, all ordinary irreducible characters of $G$ can be found except for those corresponding to the semisimple representatives $t_{7}, t_{9}$, $t_{12}$ and $t_{13}$. From the part of the character table given in CHEVIE we can read off the following:

Exactly 10 of the unipotent characters are $p$-exceptional. These are, using the notation of $[\mathbf{2 0}],\left\{\xi_{5}, \xi_{6}\right\},\left\{\xi_{7}, \xi_{8}\right\},\left\{\xi_{15}, \xi_{16}\right\},\left\{\xi_{17}, \xi_{18}\right\}$ and $\left\{\xi_{19}, \xi_{20}\right\}$. By [20, Thm. 4.6 and Thm. 4.7] and Lemma 2.14, $\sigma \alpha_{i}^{-1}$ operates trivially on these 10 unipotent characters.

Looking at the other characters listed in CHEVIE, $\left\{\chi_{23}(k), \chi_{24}(k)\right\}$ are $p$-exceptional for all parameters $k$. According to the Jordan decomposition, the characters $\chi_{22}(k)$ through $\chi_{25}(k)$ belong to the semisimple class type $t_{1}$. If $s$ is the representative of the $G$-conjugacy class of type $t_{1}$ corresponding to $k$, then $C_{G}(s) \cong \mathbb{Z}_{q^{2}-1} \times{ }^{2} B_{2}\left(q^{2}\right)$. Thus there are four possibilities for $\lambda$, namely the unipotent characters of ${ }^{2} B_{2}\left(q^{2}\right)$ : $1, \mu, \bar{\mu}$ and St. Therefore we have the correspondence $\chi_{22}(k) \leftrightarrow \chi_{s, 1}$, $\chi_{23}(k) \leftrightarrow \chi_{s, \mu}, \chi_{24}(k) \leftrightarrow \chi_{s, \bar{\mu}}$ and $\chi_{25}(k) \leftrightarrow \chi_{s, \mathrm{St}}$.

For a rational prime $l \mid\left(q^{2}-\sqrt{2} q+1\right)$, we have the following Brauer trees for such $s[\mathbf{1 9}$, Satz D.3.9]:

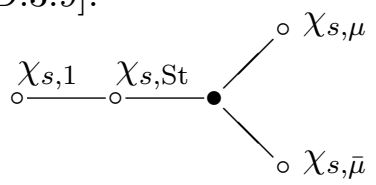

Thus, by Lemma 2.13, $\sigma \alpha_{i}^{-1}$ cannot induce the permutation $\left(\chi_{s, \mu}, \chi_{s, \bar{\mu}}\right)$ which means that $\sigma \alpha_{i}^{-1}$ cannot permute $\chi_{23}(k)$ and $\chi_{24}(k)$.

There are similar Brauer trees for the semisimple class types $t_{7}$ and $t_{9}$. We have $C_{G}\left(t_{7}\right) \cong \mathbb{Z}_{q^{2}-\sqrt{2} q+1} \times{ }^{2} B_{2}\left(q^{2}\right), C_{G}\left(t_{9}\right) \cong \mathbb{Z}_{q^{2}+\sqrt{2} q+1} \times$ ${ }^{2} B_{2}\left(q^{2}\right)$. For each $G$-conjugacy class of $s$ of type $t_{7}$ or $t_{9}$, there are again 4 characters $\chi_{s, 1}, \chi_{s, \mu}, \chi_{s, \bar{\mu}}$ and $\chi_{s, \mathrm{St}}$. The Brauer trees are then as follows:

For $s$ of type $t_{9}$ and $l \mid\left(q^{2}-\sqrt{2} q+1\right)$, we get a tree analogous to the one for $s$ of type $t_{1}$ [19, Satz D.3.9].

For $s$ of type $t_{7}$ and $l \mid\left(q^{2}+\sqrt{2} q+1\right)$, the Brauer trees look like [19, Satz D.3.10]:

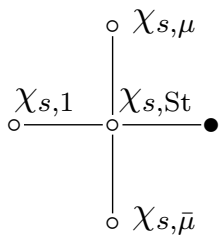


Thus, by Lemma 2.13, $\sigma \alpha_{i}^{-1}$ cannot permute $\chi_{s, \mu}$ and $\chi_{s, \bar{\mu}}$ for $s$ of type $t_{7}$ or $t_{9}$.

It remains to show that $\sigma \alpha_{i}^{-1}$ operates trivially within each row of the ordinary generic character table.

This is clear for all the characters listed in CHEVIE except for those corresponding to the semisimple class type $t_{1}$. Thus we have to look at the characters corresponding to the semisimple types $t_{1}, t_{7}, t_{9}$ and $t_{12}, t_{13}$.

Since $t_{12}$ and $t_{13}$ are regular, i.e. the corresponding characters are of the form $\pm R_{\mathbb{T}, \theta}$ with $\mathbb{T}$ of type $T(6)$ for $t_{12}$ and of type $T(7)$ for $t_{13}$ and suitable $\theta$ in general position, the claim follows almost immediately.

For $s$ of type $t=t_{1}, t_{7}$ or $t_{9}$, respectively, we look at $\chi_{s, 1}+\chi_{s, \mathrm{St}}$. If $s$ and $s^{\prime}$ belong to different $G$-conjugacy classes of type $t$, it follows that $\left.\left(\chi_{s, 1}+\chi_{s, \mathrm{St}}\right)\right|_{G_{p^{\prime}}} \neq\left.\left(\chi_{s^{\prime}, 1}+\chi_{s^{\prime}, \mathrm{St}}\right)\right|_{G_{p^{\prime}}}$. Thus it follows that either $\left.\left(\chi_{s, 1}\right)\right|_{G_{p^{\prime}}} \neq\left.\left(\chi_{s^{\prime}, 1}\right)\right|_{G_{p^{\prime}}}$ or $\left.\left(\chi_{s, \mathrm{St}}\right)\right|_{G_{p^{\prime}}} \neq\left.\left(\chi_{s^{\prime}, \mathrm{St}}\right)\right|_{G_{p^{\prime}}}$. Therefore $\sigma \alpha_{i}^{-1}$ either does not permute $\chi_{s, 1}$ and $\chi_{s^{\prime}, 1}$ or it does not permute $\chi_{s, \text { St }}$ and $\chi_{s^{\prime}, \mathrm{St}}$. Using the above Brauer trees, $\chi_{s, 1}, \chi_{s, \mu}, \chi_{s, \bar{\mu}}$ and $\chi_{s, \mathrm{St}}$ lie in the same Brauer tree of an $l$-block and are not $l$-exceptional. Thus $\sigma \alpha_{i}^{-1}$ operates trivially on the rows of the generic ordinary character table corresponding to the semisimple class type $t$.

This proves Proposition 3.1(ii).

Remark 3.2. The conjecture (ZC) is also valid for the simple Tits group ${ }^{2} F_{4}(2)^{\prime}$, which is the normal subgroup of ${ }^{2} F_{4}(2)$ of index 2 . Since the proof uses different methods from those described here, we state this result without proof.

Proof of Theorem 1. The simple groups with abelian Sylow 2-subgroups have been classified by Walter [25, XI Thm. 13.7]. These are $\operatorname{PSL}\left(2,2^{f}\right)$ for $f \geq 1, \operatorname{PSL}(2, q)$ for $q=p^{f}, q \neq 3$, with $q \equiv 3(8)$ or $q \equiv 5$ (8), the Janko group $\mathrm{J}_{1}$ and the finite Ree groups of type $G_{2}$.

The validity of $(\mathrm{ZC})$ for $\operatorname{PSL}\left(2, p^{f}\right)$ for arbitrary rational primes $p$ has been proved in [4, Prop. 3.2 and Prop. 4.1]. The theory of principal blocks with cyclic defect groups together with Lemma 2.14 shows that (ZC) holds for $\mathrm{J}_{1}$. Thus, together with Proposition 3.1(i), Theorem 1 follows.

\section{Finite groups of Lie type of rank 2 .}

In this section we want to deal with finite Chevalley groups and twisted groups of rank 2. This will especially prove Theorem 2 .

4.1. The linear and unitary groups $\operatorname{SL}(3, q), \operatorname{PSL}(3, q), \operatorname{SU}\left(3, q^{2}\right)$ and $\operatorname{PSU}\left(3, q^{2}\right)$. The groups $\mathrm{SL}(3, q)$ are simple for $q \not \equiv 1(3)$, and $\mathrm{SU}\left(3, q^{2}\right)$ are 
simple for $q \not \equiv-1(3)$. In these cases, the group automorphisms are generated by inner, graph and field automorphisms. In all other cases, there also exist diagonal automorphisms. The ordinary generic character tables have been determined in [41] and can also be found in CHEVIE. We first prove the following:

Proposition 4.1. The conjecture (ZC) is valid for $\mathrm{SL}(3, q)$ and $\mathrm{SU}\left(3, q^{2}\right)$ for all $q=p^{m}$, where $p$ is a rational prime and $m \geq 1$.

Proof. Let $G=\mathrm{SL}(3, q)$ or $G=\mathrm{SU}\left(3, q^{2}\right)$, respectively.

Part (A).

By [33, Thm. 1.1], all simple $k G$-modules with dimension smaller than or equal to 5 are of the form $M_{10}^{\alpha_{j}}$ or $M_{01}^{\alpha_{j}}$ for some $j$. Note that if $\gamma$ is the nontrivial graph automorphism for $G=\mathrm{SL}(3, q)$, and if $\gamma$ is the field automorphism $\alpha_{m}$ for $G=\mathrm{SU}\left(3, q^{2}\right)$, then $M_{10}^{\gamma} \cong M_{01}$. Furthermore, since in case $G=\mathrm{SL}(3, q), \gamma$ operates on the conjugacy classes as $(\mathrm{Cl}(g))^{\gamma}=$ $\mathrm{Cl}\left(g^{-1}\right)$ and since $\sigma$ preserves the power map, the action of $\sigma$ on the $p$ modular Brauer characters commutes with the action of $\gamma$. This implies hypothesis (i) of Proposition 2.11. The weak order $\prec$ on $X^{+}$for hypothesis (ii) is defined as follows:

Let $\mu_{1}=b_{1} b_{2}$ and $\mu_{2}=c_{1} c_{2}$ be two dominant weights. Then $\mu_{1} \prec \mu_{2}$ if and only if $b_{1}+b_{2} \leq c_{1}+c_{2}$. To show that $\prec$ is allowable, we only have to show that $\mu_{1} \leq \mu_{2}, \mu_{1} \neq \mu_{2}$ implies $\mu_{1} \prec \mu_{2}$ and $\mu_{2} \nprec \mu_{1}$. If $s_{1}$ and $s_{2}$ are the fundamental roots corresponding to 10 and 01 , then we have

$$
\begin{aligned}
& 10=\frac{1}{3}\left(2 \cdot s_{1}+s_{2}\right), \\
& 01=\frac{1}{3}\left(s_{1}+2 \cdot s_{2}\right) .
\end{aligned}
$$

Thus it follows that $\mu_{1} \leq \mu_{2}$ if and only if

$$
\begin{array}{r}
2 b_{1}+b_{2} \leq 2 c_{1}+c_{2}, \\
b_{1}+2 b_{2} \leq c_{1}+2 c_{2},
\end{array}
$$

and $2\left(c_{1}-b_{1}\right)+\left(c_{2}-b_{2}\right)$ and $\left(c_{1}-b_{1}\right)+2\left(c_{2}-b_{2}\right)$ are multiples of 3 . This implies that $b_{1}+b_{2} \leq c_{1}+c_{2}$. If $b_{1}+b_{2}=c_{1}+c_{2}$ and $\mu_{1} \neq \mu_{2}$, then $\mu_{1} \not \leq \mu_{2}$. Thus $\mu_{1} \leq \mu_{2}, \mu_{1} \neq \mu_{2}$ implies $\mu_{1} \prec \mu_{2}$ and $\mu_{2} \nprec \mu_{1}$.

Part (B).

$G$ has only $p$-exceptional characters if $q \equiv 1(3)$ and $G=\operatorname{SL}(3, q)$ or if $q \equiv-1(3)$ and $G=\mathrm{SU}\left(3, q^{2}\right)$.

Let now $G=\operatorname{SL}(3, q)$ and $q \equiv 1(3)$. In the notation of CHEVIE, there are 9 characters which are $p$-exceptional: $\left\{\chi_{6}, \chi_{7}, \chi_{8}\right\},\left\{\chi_{11}, \chi_{12}, \chi_{13}\right\}$ and $\left\{\chi_{14}, \chi_{15}, \chi_{16}\right\}$. The following actions on these characters are induced by group automorphisms: There exists a diagonal automorphism $\delta$ with operation $\tilde{\delta}=\left(\chi_{6}, \chi_{7}, \chi_{8}\right)\left(\chi_{11}, \chi_{12}, \chi_{13}\right)\left(\chi_{14}, \chi_{15}, \chi_{16}\right)$. Note that we use here cycle notation. For the graph automorphism $\gamma$ we get as operation 
$\tilde{\gamma}=\left(\chi_{11}, \chi_{14}\right)\left(\chi_{12}, \chi_{15}\right)\left(\chi_{13}, \chi_{16}\right)$. If $p \equiv 1(3)$, we get for the field automorphism $\alpha_{1}$ the same operation as for $\gamma$. We want to show that the operations on the $9 p$-exceptional characters generated by group automorphisms of $G$ are the only operations that can be induced by $\sigma$. Note that if $\chi_{11}$ and $\chi_{14}$ are permuted then this induces a nontrivial operation on the $p$-regular classes. On the other hand $\delta$ operates trivially on the $p$-regular classes. Thus we only have to show that all operations on these 9 characters induced by $\sigma$ are generated by $\pi_{1}=\left(\chi_{6}, \chi_{7}, \chi_{8}\right)\left(\chi_{11}, \chi_{12}, \chi_{13}\right)\left(\chi_{14}, \chi_{15}, \chi_{16}\right)$ and $\pi_{2}=\left(\chi_{11}, \chi_{14}\right)\left(\chi_{12}, \chi_{15}\right)\left(\chi_{13}, \chi_{16}\right)$.

Note that $|G|=q^{3}(q-1)^{2}(q+1)\left(q^{2}+q+1\right)$. By [24, II Satz 7.3], $G$ has a cyclic subgroup of order $q^{2}+q+1$. If $l \neq 3$ is a rational prime which divides $q^{2}+q+1$, then all Sylow $l$-subgroups are cyclic because $\left(q^{2}+q+1, q-1\right)=1$ and $\left(q^{2}+q+1, q+1\right)=1$. The characters $\chi_{11}$ through $\chi_{16}$ are not $l$-exceptional and lie in the following Brauer trees:
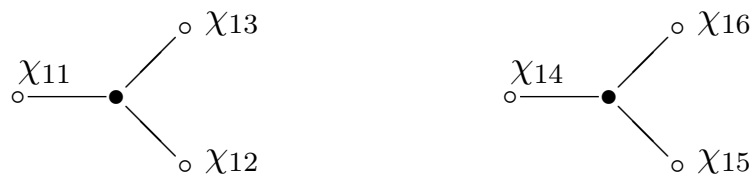

By Lemma 2.13, it follows that all operations on the characters $\chi_{11}, \chi_{12}$, $\chi_{13}, \chi_{14}, \chi_{15}, \chi_{16}$ induced by $\sigma$ are generated by $\pi_{1}$ and $\pi_{2}$. Because of the corresponding operations of $\pi_{1}$ and $\pi_{2}$ on the conjugacy classes, this also follows for the characters $\chi_{6}, \chi_{7}, \chi_{8}$.

For $G=\mathrm{SU}\left(3, q^{2}\right)$ and $q \equiv-1(3)$, the argumentation is similar. Here we use that $\mathrm{SU}\left(3, q^{2}\right)$ has a cyclic subgroup of order $q^{2}-q+1$ [32, Satz 4.2].

This proves Proposition 4.1.

For the groups $\operatorname{PSL}(3, q)$ and $\operatorname{PSU}\left(3, q^{2}\right)$ we get a similar result. Note that $\operatorname{PSL}(3, q)$ and $\operatorname{PSU}\left(3, q^{2}\right)$ are simple except for $\operatorname{PSU}\left(3,2^{2}\right) \cong\left(\mathbb{Z}_{3} \times \mathbb{Z}_{3}\right) \cdot Q_{8}$.

Proposition 4.2. The conjecture (ZC) is valid for $\operatorname{PSL}(3, q)$ and $\operatorname{PSU}\left(3, q^{2}\right)$ for all $q=p^{m}$, where $p$ is a rational prime and $m \geq 1$.

Proof. Let $\bar{G}=\operatorname{PSL}(3, q)$ or $\bar{G}=\operatorname{PSU}\left(3, q^{2}\right)$, respectively. Let $\bar{\gamma}$ be the nontrivial graph automorphism if $\bar{G}=\operatorname{PSL}(3, q)$, and $\bar{\gamma}=\bar{\alpha}_{m}$ if $\bar{G}=\operatorname{PSU}\left(3, q^{2}\right)$. Since we can use the same argumentation as in Proposition 4.1 to deal with the characters which are $p$-exceptional, we only have to look at:

Part (A).

The modules $M_{\lambda}, \lambda=a_{1} a_{2} \in X_{q}$, are simple $k \bar{G}$-modules if and only if $a_{1}+2 a_{2} \equiv 0$ (3). This follows by looking at the center of $\operatorname{SL}(3, q)$ or $\operatorname{SU}\left(3, q^{2}\right)$ as described in $[7, \S 12.1]$.

Let now $\lambda=a_{1} a_{2}$ with $a_{1}+2 a_{2} \equiv 0(3)$. Then $\lambda=a_{1} \cdot 11+\frac{1}{3}\left(a_{2}-a_{1}\right) \cdot 03$ if $a_{1} \leq a_{2}$ and $\lambda=a_{2} \cdot 11+\frac{1}{3}\left(a_{1}-a_{2}\right) \cdot 30$ if $a_{2} \leq a_{1}$. Note that $\mid a_{2}-$ $a_{1}\left|=\frac{1}{2}\right| a_{1}+2 a_{2}-3 a_{1} \mid$ is divisible by 3 . We have to show that there exists $\alpha \in \operatorname{Aut}(\bar{G})$ with $M_{11}^{\bar{\sigma}} \cong M_{11}^{\alpha}$ and $M_{03}^{\bar{\sigma}} \cong M_{03}^{\alpha}$. This then implies hypothesis 
(i) of Proposition 2.11. For hypothesis (ii) we can use the same weak order $\prec$ as for $\mathrm{SL}(3, q)$ and $\mathrm{SU}\left(3, q^{2}\right)$.

By [2, Thm. 1], all modules of dimension 8 are of the form $M_{11}^{\bar{\alpha}_{i}}$ for some $0 \leq i \leq m-1$ and, for $p>3$, all modules of dimension 10 are of the form $M_{03}^{\bar{\gamma}^{\nu} \bar{\alpha}_{j}}$ for some $\nu \in\{0,1\}$ and some $0 \leq j \leq m-1$.

Let now $p>3$. Then we get that $M_{11}^{\bar{\sigma}} \cong M_{11}^{\bar{\alpha}_{i}}$ and $M_{03}^{\bar{\sigma}} \cong M_{03}^{\bar{\gamma}^{\nu} \bar{\alpha}_{j}}$. Note that $M_{11}^{\bar{\gamma}} \cong M_{11}$. Thus we have to show that $i=j$. This follows, similarly as in the case of ${ }^{2} F_{4}\left(q^{2}\right)$, from the fact that $M_{11} \otimes M_{03}$ and $M_{11} \otimes M_{30}$ are not simple. Note that they have both dimension 80 whereas $M_{14}$ and $M_{41}$ have both dimension $\leq 35$ by Weyl's formula (see $[\mathbf{2 1}, \S 24.3]$ ). Thus we get the desired operation of $\bar{\sigma}$ on these modules.

For $p=2$, the situation is slightly more complicated. Again $M_{11}^{\bar{\sigma}} \cong M_{11}^{\bar{\alpha}_{i}}$ for some $i$. But in this case $M_{03} \cong M_{01} \otimes M_{01}^{\bar{\alpha}_{1}}$ and has dimension 9 . We have only to show that $M_{03}^{\bar{\sigma}} \cong M_{03}^{\bar{\gamma}^{\nu} \bar{\alpha}_{j}}$ for some $\nu$ and some $j$. The argumentation that $i=j$ is then similar to the case $p>3$.

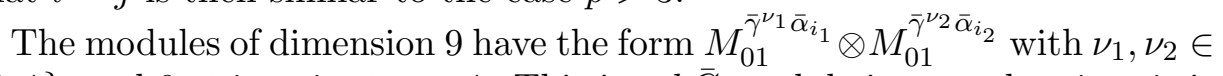
$\{0,1\}$, and $0 \leq i_{1}<i_{2} \leq m-1$. This is a $k \bar{G}$-module in case that $i_{2}-i_{1}$ is odd for $\nu_{1}=\nu_{2}$ and $i_{2}-i_{1}$ is even for $\nu_{1} \neq \nu_{2}$. For the twist of $M_{03}$ under $\bar{\sigma}$ we have to show that either $i_{2}=i_{1}+1$ and $\nu_{1}=\nu_{2}$ or $\left(i_{1}, i_{2}\right)=(0, m-1)$ and $\left(\nu_{1}=\nu_{2}\right.$ if $\bar{G}=\operatorname{PSL}(3, q)$ or $\nu_{1} \neq \nu_{2}$ if $\left.\bar{G}=\operatorname{PSU}\left(3, q^{2}\right)\right)$. Note that for $m=2, M_{03}$ and $M_{30}$ are the only $k \bar{G}$-modules of dimension 9 . So let $m \geq 3$.

Suppose first that $\nu_{1} \neq \nu_{2}$ and, if $\bar{G}=\operatorname{PSU}\left(3, q^{2}\right)$, that $\left(i_{1}, i_{2}\right) \neq(0, m-1)$. If $i_{2}=i_{1}+1$ then $i_{2}-i_{1}$ is odd, thus the module is not a $k \bar{G}$-module. So $i_{2} \neq i_{1}+1$. Then there exists $s \in\left\{i_{1}-i, i_{2}-i\right\}$ such that $s \not \equiv 0(m)$ and $s \not \equiv 1(m)$. Thus $M_{03} \otimes M_{11}^{\bar{\alpha}_{s}} \cong M_{01} \otimes M_{01}^{\bar{\alpha}_{1}} \otimes M_{11}^{\bar{\alpha}_{s}}$ is a simple module which would be mapped by $\bar{\sigma}$ to $M_{01}^{\bar{\gamma}^{\nu} \bar{\alpha}_{i_{1}}} \otimes M_{01}^{\bar{\gamma}^{\nu} \bar{\alpha}_{i_{2}}} \otimes M_{11}^{\bar{\alpha}_{i+s}}$. But this module is not simple, because neither $M_{01} \otimes M_{11}$ nor $M_{10} \otimes M_{11}$ are simple.

Suppose now that $\nu_{1}=\nu_{2}$ and $i_{2} \neq i_{1}+1$ and, if $\bar{G}=\operatorname{PSL}\left(3, q^{2}\right)$, that $\left(i_{1}, i_{2}\right) \neq(0, m-1)$. Again there exists $s \in\left\{i_{1}-i, i_{2}-i\right\}$ such that $s \not \equiv 0(m)$ and $s \not \equiv 1(m)$. So we can argue in the same way as in the first case.

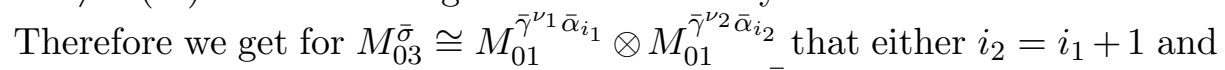
$\nu_{1}=\nu_{2}$ or $\left(i_{1}, i_{2}\right)=(0, m-1)$ and $\left(\nu_{1}=\nu_{2}\right.$ if $\bar{G}=\operatorname{PSL}(3, q)$ or $\nu_{1} \neq \nu_{2}$ if $\left.\bar{G}=\operatorname{PSU}\left(3, q^{2}\right)\right)$.

This proves Proposition 4.2.

4.2. The symplectic groups $\operatorname{Sp}(4, q)$ and $\operatorname{PSp}(4, q)$. After the linear and unitary groups of type $A_{2}$, we consider now the symplectic groups $\operatorname{Sp}(4, q)$ and $\operatorname{PSp}(4, q)$. Note that $\operatorname{Sp}\left(4,2^{m}\right)$ is simple for $m \geq 2$, and $\operatorname{Sp}(4,2)$ is isomorphic to the symmetric group on 6 elements. For even $q$, the group automorphisms are generated by inner, graph and field automorphisms. In all other cases, there exist also diagonal automorphisms, but no nontrivial 
graph automorphisms. The ordinary generic character table for even $q$ has been determined in [14] and appears also in CHEVIE. For odd $q$, the generic character table was first established by Srinivasan [42], Przygocki [34] found some small mistakes; a preliminary version computed by Lübeck in CHEVIE shows that the following corrections to Przygocki's version have to be made: For the characters $\xi_{41}^{\prime},(1-q)^{2}$ must be replaced by $\left(1-q^{2}\right)$ on the classes $D_{1}$, for $\phi_{3}$ the sign must be changed on $C_{21}$, and for $\phi_{7}$ the sign has to be changed on $B_{3}$.

We first prove the following proposition:

Proposition 4.3. The conjecture $(\mathrm{ZC})$ is valid for $\operatorname{Sp}(4, q)$ for all $q=p^{m}$ with $p$ a rational prime and $m \geq 1$.

Proof. Let $G=\operatorname{Sp}(4, q)$.

Part (A).

If $p=2$, then $X_{p}=\{00,01,10,11\}$ with $\operatorname{dim}\left(M_{01}\right)=4=\operatorname{dim}\left(M_{10}\right)$ and $M_{11} \cong M_{01} \otimes M_{10}$. If $\gamma$ denotes the nontrivial graph automorphism of $G$, then $M_{10} \cong M_{01}^{\gamma}$. By [10, Thm. (3.4)] it follows that

$$
\beta_{01} \otimes \beta_{01}=4 \cdot 1+2 \cdot \beta_{10}+\beta_{01}^{\alpha_{1}} .
$$

Because of dimensions, $\beta_{01}^{\sigma}=\beta_{01}^{\gamma^{\nu}} \alpha_{i}$ for some $\nu \in\{0,1\}, 0 \leq i \leq m-1$. Thus we get with Equation (4.1)

$$
4 \cdot 1+2 \cdot \beta_{10}^{\gamma^{\nu} \alpha_{i}}+\beta_{01}^{\gamma^{\nu} \alpha_{i+1}}=4 \cdot 1+2 \cdot \beta_{10}^{\sigma}+\beta_{01}^{\sigma \alpha_{1}}
$$

and thus $\beta_{10}^{\sigma}=\beta_{10}^{\gamma^{\nu}} \alpha_{i}$. So hypothesis (i) of Proposition 2.11 follows for the case $p=2$.

Now let $p \neq 2$. Then $\operatorname{dim}\left(M_{01}\right)=4$ and $\operatorname{dim}\left(M_{10}\right)=5$. If $M$ is a simple $k G$-module with $1<\operatorname{dim}(M)<8$ then there exists an automorphism $\alpha$ of $G$, such that either $M \cong M_{01}^{\alpha}$ or $M \cong M_{10}^{\alpha}$ [33, Thm. 1.1]. This implies hypothesis (i) in case $p \neq 2$.

The weak order $\prec$ for hypothesis (ii) is defined as follows:

Let $\mu_{1}=b_{1} b_{2}$ and $\mu_{2}=c_{1} c_{2}$ be two different dominant weights. Then $\mu_{1} \prec \mu_{2}$ if and only if $b_{1}+b_{2}<c_{1}+c_{2}$, or $b_{1}+b_{2}=c_{1}+c_{2}$ and $b_{1}<c_{1}$. Similarly to $\operatorname{SL}(3, q)$, it follows that $\mu_{1} \leq \mu_{2}, \mu_{1} \neq \mu_{2}$ implies $\mu_{1} \prec \mu_{2}$ and $\mu_{2} \nprec \mu_{1}$.

Part (B).

When $p=2, G$ has no $p$-exceptional characters. Therefore let $p \neq 2$.

We use here the notation given in $[42]$ and [34]. The characters which are $p$-exceptional are exactly the following pairs: $\left\{\xi_{21}(k), \xi_{22}(k)\right\}$ for each $k,\left\{\xi_{21}^{\prime}(k), \xi_{22}^{\prime}(k)\right\}$ for each $k,\left\{\xi_{41}(k), \xi_{42}(k)\right\}$ for each $k,\left\{\xi_{41}^{\prime}(k), \xi_{42}^{\prime}(k)\right\}$ for each $k,\left\{\Phi_{1}, \Phi_{2}\right\},\left\{\Phi_{3}, \Phi_{4}\right\},\left\{\Phi_{5}, \Phi_{6}\right\},\left\{\Phi_{7}, \Phi_{8}\right\},\left\{\theta_{1}, \theta_{2}\right\},\left\{\theta_{3}, \theta_{4}\right\},\left\{\theta_{5}, \theta_{6}\right\}$ and $\left\{\theta_{7}, \theta_{8}\right\}$. The nontrivial diagonal automorphism $\delta$ of $G$ permutes simultaneously the two characters of each such pair. Looking at the corresponding 
conjugacy classes it follows that the only possible nontrivial operation induced by $\sigma$ on these pairs is the simultaneous permutation of each pair.

This proves Proposition 4.3.

For the groups $\operatorname{PSp}(4, q)$ we get a similar result:

Proposition 4.4. The conjecture $(\mathrm{ZC})$ is valid for $\operatorname{PSp}(4, q)$ for all $q=p^{m}$ with $p$ a rational prime and $m \geq 1$.

Proof. Let $\bar{G}=\operatorname{PSp}(4, q)$. Because of Proposition 4.3, we only have to show Proposition 4.4 for $p \geq 3$. So let $p \geq 3$. Since we can use the same argumentation as in Proposition 4.3 to deal with the characters which are exceptional for $p$, we only have to look at:

Part (A).

The modules $M_{\lambda}, \lambda=a_{1} a_{2} \in X_{q}$, are simple $k \bar{G}$-modules if and only if $a_{2} \equiv 0(2)$. This follows by looking at the center of $\operatorname{Sp}(4, q)$ as described in $[7, \S 12.1]$.

Let now $\lambda=a_{1} \lambda_{1}+a_{2} \lambda_{2}=a_{1} a_{2}$ with $a_{2} \equiv 0(2)$. Then $\lambda=a_{1} \cdot 10+\frac{1}{2} a_{2} \cdot 02$. We have to show that there exists $\alpha \in \operatorname{Aut}(\bar{G})$ with $M_{10}^{\bar{\sigma}} \cong M_{10}^{\alpha}$ and $M_{20}^{\bar{\sigma}} \cong$ $M_{20}^{\alpha}$. This then implies hypothesis (i) of Proposition 2.11. For hypothesis (ii) we can use the same weak order $\prec$ as for $\operatorname{Sp}(4, q)$.

By [2, Thm. 1], all modules of dimension 5 are of the form $M_{10}^{\bar{\alpha}_{i}}$ for some $i$, and all modules of dimension 10 are of the form $M_{02}^{\bar{\alpha}_{j}}$ for some $j$. Thus we get the desired operation of $\bar{\sigma}$ on these modules.

This proves Proposition 4.4.

4.3. The finite Chevalley groups $G_{2}(q)$. After the classical groups of type $A_{2}$ and $B_{2}$, we want now to examine the exceptional groups of type $G_{2}$. The ordinary generic character tables of these groups can be found in $[\mathbf{1 5}]$ and in $[\mathbf{9}, \mathbf{1 6}]$ and have also been determined in CHEVIE.

$G_{2}(q)$ is simple except for $G_{2}(2) \cong \mathrm{SU}\left(3,3^{2}\right) \cdot 2=\operatorname{Aut}\left(\mathrm{SU}\left(3,3^{2}\right)\right)$. For $3 / q$, the group automorphisms of $G_{2}(q)$ are generated by inner and field automorphisms. For $q=3^{m}$ there exists also a nontrivial graph automorphism of order 2 .

We want to prove the following proposition:

Proposition 4.5. The conjecture $(\mathrm{ZC})$ is valid for $G_{2}(q)$ for all $q=p^{m}$ with $p$ a rational prime and $m \geq 1$.

Proof. Let $G=G_{2}(q)$.

Part (A).

If $p=3$, then $X_{p}=\{00,01,10,02,20,11,12,21,22\}$ with $\operatorname{dim}\left(M_{01}\right)=$ $7=\operatorname{dim}\left(M_{10}\right), \operatorname{dim}\left(M_{02}\right)=27=\operatorname{dim}\left(M_{20}\right), M_{11} \cong M_{01} \otimes M_{10}, M_{22} \cong$ $M_{02} \otimes M_{20}, M_{12} \cong M_{02} \otimes M_{10}$ and $M_{21} \cong M_{01} \otimes M_{20}$. If $\gamma$ denotes the nontrivial graph automorphism of $G$, then $M_{10} \cong M_{01}^{\gamma}, M_{20} \cong M_{02}^{\gamma}$ and 
$M_{12} \cong M_{21}^{\gamma}$. Using the 3 -modular character table of $G_{2}(3)$ listed in GAP [39], it follows that for $q=3$ the tensor product $\beta_{01} \otimes \beta_{01}$ decomposes as

$$
\beta_{01} \otimes \beta_{01}=1 \cdot 1+2 \cdot \beta_{01}+\beta_{10}+\beta_{02} \text {. }
$$

This is valid for arbitrary $q=3^{m}$, since the weights $\lambda \in X^{+}$with $\lambda \leq 02$ are exactly $\lambda \in\{00,01,10,02\}$.

Because of dimensions, $\beta_{01}^{\sigma}=\beta_{01}^{\gamma^{\nu} \alpha_{i}}$ for some $\nu \in\{0,1\}, 0 \leq i \leq m-1$. Thus we get with Equation (4.2)

$$
1 \cdot 1+2 \cdot \beta_{01}^{\gamma^{\nu} \alpha_{i}}+\beta_{10}^{\gamma^{\nu} \alpha_{i}}+\beta_{02}^{\gamma^{\nu} \alpha_{i}}=1 \cdot 1+2 \cdot \beta_{01}^{\sigma}+\beta_{10}^{\sigma}+\beta_{02}^{\sigma}
$$

and therefore

$$
\beta_{10}^{\gamma^{\nu} \alpha_{i}}+\beta_{02}^{\gamma^{\nu} \alpha_{i}}=\beta_{10}^{\sigma}+\beta_{02}^{\sigma} .
$$

Since the Brauer characters are linearly independent, it follows because of different dimensions that $\beta_{10}^{\sigma}=\beta_{10}^{\gamma^{\nu} \alpha_{i}}$ and $\beta_{02}^{\sigma}=\beta_{02}^{\gamma^{\nu} \alpha_{i}}$. Thus hypothesis (i) of Proposition 2.11 follows for the case $p=3$.

Now let $p \neq 3$. Then $\operatorname{dim}\left(M_{01}\right)=7-\delta_{p, 2}$ and $\operatorname{dim}\left(M_{10}\right)=14$. If $M$ is a simple $k G$-module with $1<\operatorname{dim}(M)<18$ then there exists an automorphism $\alpha$ of $G$, such that either $M \cong M_{01}^{\alpha}$ or $M \cong M_{10}^{\alpha}$. This follows from [31, Prop. 5.4.12], since the center of $G_{2}(q)$ is trivial and thus every simple $k G$-module is also a simple projective $k G$-module. Therefore, hypothesis (i) follows for $p \neq 3$.

For the weak order $\prec$ in hypothesis (ii) we take the natural order relation $\leq$

Part (B).

We have to show that if $\alpha$ is the group automorphism from Part (A), then $\sigma \alpha^{-1}$ operates trivially on the characters which are $p$-exceptional.

In CHEVIE, the ordinary generic character tables of $G_{2}(q)$ are divided into five cases. In all five cases, only the characters $\chi_{8}$ and $\chi_{9}$ are $p$ exceptional.

Note that $|G|=q^{6}(q-1)^{2}(q+1)^{2}\left(q^{2}-q+1\right)\left(q^{2}+q+1\right)$, and that $G$ has maximal tori $H_{3} \cong \mathbb{Z}_{q^{2}+q+1}$ and $H_{6} \cong \mathbb{Z}_{q^{2}-q+1}$.

$q \equiv 1(3):$

Let $l$ be a rational prime dividing $q^{2}-q+1$. Because of $\left(q^{2}-q+1, q-1\right)=1$, $\left(q^{2}-q+1, q+1\right)=1$ and $\left(q^{2}-q+1, q^{2}+q+1\right)=1$, a Sylow $l$-subgroup of $H_{6}$ is also a Sylow $l$-subgroup of $G$, i.e. all Sylow $l$-subgroups of $G$ are cyclic. Since $\chi_{8}$ and $\chi_{9}$ belong to the principal $l$-block and are not $l$-exceptional, $\sigma \alpha^{-1}$ fixes $\chi_{8}$ and $\chi_{9}$ by Lemma 2.14 .

$q \equiv-1(3)$ or $q \equiv 0(3)$ :

Let $l$ be a rational prime dividing $q^{2}+q+1$. Similarly to $q \equiv 1(3)$ it follows that all Sylow $l$-subgroups of $G$ are cyclic. $\chi_{8}$ and $\chi_{9}$ belong to the principal $l$-block and are not $l$-exceptional. Thus by Lemma $2.14, \sigma \alpha^{-1}$ fixes $\chi_{8}$ and $\chi_{9}$. 
This proves Proposition 4.5.

4.4. The finite twisted groups ${ }^{3} D_{4}\left(q^{3}\right)$. The finite groups of type ${ }^{3} D_{4}$ are the only missing simple exceptional groups of rank $2 .{ }^{3} D_{4}\left(q^{3}\right)$ is simple for all $q$, and all group automorphisms are generated by inner and field automorphisms.

The generic character tables of ${ }^{3} D_{4}\left(q^{3}\right)$ have been determined in [13] and can also be found in CHEVIE.

Proposition 4.6. The conjecture (ZC) is valid for ${ }^{3} D_{4}\left(q^{3}\right)$ for all $q=p^{m}$, $p$ a rational prime and $m \geq 1$.

Proof. Let $G={ }^{3} D_{4}\left(q^{3}\right)$. Since there exist no ordinary irreducible characters of $G$ which are $p$-exceptional, it suffices to look at:

Part (A).

The simple modules $M_{\lambda_{i}}$ satisfy $\operatorname{dim}\left(M_{\lambda_{1}}\right)=8$ and $\operatorname{dim}\left(M_{\lambda_{2}}\right)=28-2 \delta_{p, 2}$, $M_{\lambda_{3}} \cong M_{\lambda_{1}}^{\alpha_{m}}$ and $M_{\lambda_{4}} \cong M_{\lambda_{1}}^{\alpha_{2 m}}$. By [33, Thm. 1.1], for every simple $k G$ module $M$ with $1<\operatorname{dim}(M)<32$ there exists a group automorphism $\alpha$ of $G$ such that either $M \cong M_{\lambda_{1}}^{\alpha}$ or $M \cong M_{\lambda_{2}}^{\alpha}$. Note that [33, Thm. 1.1] shows this for the finite Chevalley groups of type $D_{4}$, but by Theorem 2.4, this must also be valid for $G$. This implies hypothesis (i) of Proposition 2.11. The weak order $\prec$ for hypothesis (ii) is defined as follows:

Let $\mu_{1}=b_{1} b_{2} b_{3} b_{4}$ and $\mu_{2}=c_{1} c_{2} c_{3} c_{4}$ be two different dominant weights. Then $\mu_{1} \prec \mu_{2}$ if and only if $b_{1}+\frac{3}{2} b_{2}+b_{3}+b_{4}<c_{1}+\frac{3}{2} c_{2}+c_{3}+c_{4}$, or $b_{1}+\frac{3}{2} b_{2}+b_{3}+b_{4}=c_{1}+\frac{3}{2} c_{2}+c_{3}+c_{4}$ and $b_{2}<c_{2}$. Similarly to the case $\mathrm{SL}(3, q)$, it follows that $\mu_{1} \leq \mu_{2}, \mu_{1} \neq \mu_{2}$ implies $\mu_{1} \prec \mu_{2}$ and $\mu_{2} \nprec \mu_{1}$.

This proves Proposition 4.6.

The Propositions 3.1, 4.2, 4.4, 4.5 and 4.6 together with the results of [4] prove Theorem 2.

Altogether the conjecture (ZC) is valid for the following finite groups of Lie type:

$$
\begin{gathered}
\mathrm{SL}\left(2, p^{m}\right), \operatorname{PSL}\left(2, p^{m}\right), p \text { a rational prime } \\
{ }^{2} B_{2}\left(2^{2 m+1}\right),{ }^{2} G_{2}\left(3^{2 m+1}\right),{ }^{2} F_{4}\left(2^{2 m+1}\right) \\
\mathrm{SL}\left(3, p^{m}\right), \operatorname{PSL}\left(3, p^{m}\right), p \text { a rational prime } \\
\mathrm{SU}\left(3, p^{2 m}\right), \operatorname{PSU}\left(3, p^{2 m}\right), p \text { a rational prime } \\
\mathrm{Sp}\left(4, p^{m}\right), \operatorname{PSp}\left(4, p^{m}\right), p \text { a rational prime } \\
G_{2}\left(p^{m}\right), p \text { a rational prime } \\
{ }^{3} D_{4}\left(p^{3 m}\right), p \text { a rational prime. }
\end{gathered}
$$


Acknowledgments. The results of this paper except for the results concerning the series of type $A_{2},{ }^{2} A_{2}$ and $B_{2}$ originate in my Ph.D. thesis [3]. I would like to express my thanks to Prof. Dr. W. Kimmerle and Prof. Dr. K.W. Roggenkamp for their continuous support.

\section{References}

[1] J.L. Alperin, Local representation theory, Cambridge studies in advanced mathematics 11, Cambridge University Press, 1986.

[2] M. Aschbacher, Small degree representations of groups of Lie type, preprint, 1987.

[3] F.M. Bleher, Automorphismen von Gruppenringen und Blocktheorie, Dissertation, Stuttgart, 1995.

[4] _ Tensor products and a conjecture of Zassenhaus, Archiv d. Math., 64 (1995), 289-298.

[5] _ Integral group rings of finite groups of Lie type, to appear.

[6] F.M. Bleher, G. Hiss and W. Kimmerle, Autoequivalences and a conjecture of Zassenhaus, J. Pure and Appl. Alg., 103 (1995), 23-43.

[7] R.W. Carter, Simple Groups of Lie Type, John Wiley \& Sons, New York, 1972.

[8] Finite Groups of Lie Type: Conjugacy Classes and Complex Characters, John Wiley \& Sons, New York, 1985.

[9] B. Chang and R. Ree. The characters of $G_{2}(q)$, in 'Symposia Mathematica', Vol. 13, Academic Press, London, (1974), 395-413.

[10] L. Chastkofsky and W.Feit, On the projective characters in characteristic 2 of the groups Suz $\left(2^{m}\right)$ and $S p_{4}\left(2^{n}\right)$, Inst. Hautes Etudes Sci. Publ. Math., 51 (1980), 9-35.

[11] R. Curtis and I. Reiner, Methods of Representation Theory, Vol. I, II, John Wiley \& Sons, New York, 1981, 1987.

[12] E.C. Dade, Bocks with cyclic defect groups, Ann. of Math., 84 (1966), 20-48.

[13] D.I. Deriziotis and G.O. Michler, Character table and blocks of finite simple triality groups ${ }^{3} D_{4}(q)$, Trans. Am. Math. Soc., 303 (1987), 39-70.

[14] H. Enomoto, The characters of the finite symplectic group $\operatorname{Sp}(4, q), q=2^{f}$, Osaka J. Math., 9 (1972), 75-94.

[15] - The characters of the finite Chevalley group $G_{2}(q), q=3^{f}$, Japan. J. Math., 2 (1976), 191-248.

[16] H. Enomoto and H. Yamada, The characters of $G_{2}\left(2^{n}\right)$, Japan. J. Math., 12 (1986), 325-377.

[17] M. Geck, G. Hiss, F. Lübeck, G. Malle and G. Pfeiffer, CHEVIE - Generic Character Tables of Finite Groups of Lie Type, Hecke Algebras and Weyl Groups, IWR-Preprint, 93-62, Heidelberg, 1993.

[18] M. Hertweck, Two non-isomorphic finite groups with isomorphic integral group rings, preprint, 1997.

[19] G. Hiss, Zerlegungszahlen endlicher Gruppen vom Lie-Typ in nichtdefinierender Charakteristik, Habilitationsschrift, Aachen, 1990.

[20] , The Brauer trees of the Ree groups, Comm. in Alg., 19(3) (1991), 871-888. 
[21] J.E. Humphreys, Introduction to Lie Algebras and Representation Theory, Graduate Texts in Math., 9, Springer-Verlag, New York, 1972.

[22] _ Linear Algebraic Groups, Graduate Texts in Math., 21, Springer-Verlag, New York, 1975.

[23] _ Ordinary and Modular Representations of Chevalley Groups, Lecture Notes in Math., 528, Springer-Verlag, New York, 1976.

[24] B. Huppert, Endliche Gruppen I, Grundlehren der mathematischen Wissenschaften, 134, Springer-Verlag, Berlin, 1967.

[25] B. Huppert and N. Blackburn, Finite Groups III, Grundlehren der mathematischen Wissenschaften, 243, Springer-Verlag, Berlin, 1982.

[26] N. Kawanaka, Generalized Gelfand-Graev representations and Ennola duality, in 'Algebraic Groups and Related Topics', Adv. Stud. Pure Math., 6, North Holland, Amsterdam, (1985), 175-206.

[27] W. Kimmerle, Beiträge zur ganzzahligen Darstellungstheorie endlicher Gruppen, Bayreuther Mathematische Schriften, Heft 36, 1991.

[28] - More on the class sum correspondence, in 'Group Rings and Class Groups', DMV Seminar Band, 18, Birkhäuser Verlag, Basel, (1992), 27-37.

[29] W. Kimmerle, R. Lyons, R. Sandling and D. Teague, Composition factors from the group ring and Artin's theorem on orders of simple groups, Proc. London Math. Soc., 60(3) (1990), 89-122.

[30] W. Kimmerle and K.W. Roggenkamp, Projective limits of group rings, J. Pure Appl. Algebra, 88 (1993), 119-142.

[31] P. Kleidman and M. Liebeck, The Subgroup Structure of the Finite Classical Groups, London Mathematical Society Lecture Note Series 129, Cambridge University Press, Cambridge, 1990.

[32] M. Klemm, Charakterisierung der Gruppen $\operatorname{PSL}\left(2, p^{f}\right)$ und $\operatorname{PSU}\left(3, p^{2 f}\right)$ durch ihre Charaktertafel, J. Algebra, 24 (1973), 127-153.

[33] M.W. Liebeck, On the orders of maximal subgroups of the finite classical groups, Proc. London Math. Soc., 50(3) (1985), 426-446.

[34] A. Przygocki, Schur indices of symplectic groups, Comm. in Alg., 10(3) (1982), 279310 .

[35] K.W. Roggenkamp, The isomorphism problem for integral group rings of finite groups, Proceedings of the International Congress of Mathematicians Kyoto 1990, SpringerVerlag, Tokyo, (1991), 369-380.

[36] K.W. Roggenkamp and L.L. Scott, Isomorphisms of p-adic group rings, Ann. of Math., 126 (1987), 593-647.

[37] _ A strong answer to the isomorphism problem for finite p-solvable groups with a normal p-subgroup containing its centralizer, preprint, 1987.

[38] K.W. Roggenkamp and M.J. Taylor, Group Rings and Class Groups, DMV Seminar Band 18, Birkhäuser Verlag, Basel, 1992.

[39] M. Schönert et al, GAP - Groups, Algorithms and Programming, Lehrstuhl D für Mathematik, RWTH Aachen, Germany, first ed. 1992.

[40] K. Shinoda, The conjugacy classes of the finite Ree groups of type $\left(F_{4}\right)$, J. Fac. Sci. Univ. Tokyo Sec. IA, 22 (1975), 1-15. 
[41] W.A. Simpson and J.S. Frame, The character tables for $\mathrm{SL}(3, q), \mathrm{SU}\left(3, q^{2}\right), \operatorname{PSL}(3, q)$, $\operatorname{PSU}\left(3, q^{2}\right)$, Canad. J. Math., 25(I) (1973), 486-494.

[42] B. Srinivasan, The characters of the finite symplectic group $\operatorname{Sp}(4, q)$, Trans. Amer. Math. Soc., 131 (1968), 488-525.

[43] R. Steinberg, Representations of algebraic groups, Nagoya Math. J., 22 (1963), 33-56.

[44] H.J. Ward, On Ree's series of simple groups, Trans. Amer. Math. Soc., 121 (1966), 62-89.

[45] A. Weiss, p-adic rigidity of p-torsion, Ann. of Math., 127(2) (1987), 317-332.

[46] _ Torsion units in integral group rings, J. Reine Angew. Math., 415 (1991), 175-187.

Received August 21, 1997 and revised February 9, 1998.

SOUTHERN ILLINOIS UNIVERSITY

Carbondale, IL 62901-4408

E-mail address: fbleher@math.siu.edu 